\title{
Low Overhead Broadcast Encryption from Multilinear Maps
}

\author{
Dan Boneh $^{1}$, Brent Waters ${ }^{2}$, and Mark Zhandry ${ }^{1}$ \\ 1 Stanford University, CA, USA \\ \{dabo, zhandry\}@cs.stanford.edu \\ 2 University of Texas at Austin, TX, USA \\ bwaters@cs.utexas.edu
}

\begin{abstract}
We use multilinear maps to provide a solution to the longstanding problem of public-key broadcast encryption where all parameters in the system are small. In our constructions, ciphertext overhead, private key size, and public key size are all poly-logarithmic in the total number of users. The systems are fully collusion-resistant against any number of colluders. All our systems are based on an $O(\log N)$-way multilinear map to support a broadcast system for $N$ users. We present three constructions based on different types of multilinear maps and providing different security guarantees. Our systems naturally give identity-based broadcast systems with short parameters.
\end{abstract}

\section{Introduction}

Broadcast encryption [FN94] is an important generalization of public-key encryption to the multi-user setting. In a broadcast encryption scheme, a broadcaster encrypts a message for a subset $S$ of users who are listening on a broadcast channel. The broadcaster can encrypt to any set $S$ of its choice, and any user in $S$ can decrypt the broadcast using its secret key. The system is said to be fully collusion resistant if even a coalition of all users outside of $S$ learns nothing about the plaintext. Broadcast systems are regularly used in TV and radio subscription services where broadcasts are encrypted for currently active subscribers. They are also used in encrypted file systems where a file is encrypted so that only users who have access to the file can decrypt it.

The efficiency of a broadcast system is measured in the ciphertext overhead: the number of bits in the ciphertext beyond what is needed for the description of the recipient set $S$ and the symmetric encryption of the plaintext payload. The shorter the overhead, the better. We say that the system has low overhead if the ciphertext overhead depends at most logarithmically on the number of users $N$ in the system.

Existing constructions with low ciphertext overhead. Several broadcast systems are fully collusion resistant with low ciphertext overhead. The first such system by Boneh, Gentry, and Waters BGW05] is built from bilinear maps. It has

J.A. Garay and R. Gennaro (Eds.): CRYPTO 2014, Part I, LNCS 8616, pp. 206-223, 2014.

(C) International Association for Cryptologic Research 2014 
constant ciphertext overhead and short secret keys, but the public encryption key size is linear in the number of users $N$. Other systems using bilinear-maps achieve adaptive security GW09, DPP07 and some are even identity-based GW09. Del07, SF07, but the public encryption key is always large.

Multilinear maps give secret-key broadcast systems with optimal ciphertext overhead [BS03, GGH13a, FHPS13, CLT13, BW13]. However, in these systems the broadcaster's key must be kept secret, and they require an $N$-way multilinear map to support $N$ users. Current constructions of $N$-linear maps GGH13a, CLT13 have group elements of size $O\left(N^{2}\right)$ bits, resulting in large space requirements. While these broadcast systems can be made public-key by including a few group elements in the ciphertext, their dependence on $N$-linear maps leads to an $O\left(N^{2}\right)$ ciphertext overhead, which is worse than the trivial broadcast system. Until this work, it has not been known how to use multilinear maps to construct low overhead broadcast systems with a short public encryption key.

A third class of constructions employs the powerful candidates for indistinguishability obfuscation (iO) $\left[\mathrm{BGI}^{+} 01, \mathrm{GGH}^{+} 13 \mathrm{~b}\right]$. Using $\mathrm{iO}$ it is possible to build a public-key broadcast system with optimal ciphertext overhead and short private keys, though public keys are large [BZ14. The resulting systems have several other remarkable properties. However, current iO candidates add considerable complexity on top of multilinear maps. Our goal here is to construct broadcast systems using only simple assumptions on multilinear maps, namely, without relying on $\mathrm{iO}$.

Our results. We describe three broadcast systems for $N$ users that use an $O(\log N)$-way multilinear map. The systems have ciphertext overhead and decryption key of only $O(1)$ group elements which is $O\left(\log ^{2} N\right)$ bits using the current multilinear map candidates. The public encryption key contains $O(\log N)$ group elements which is $O\left(\log ^{3} N\right)$ bits. The first system uses an asymmetric multilinear map and follows the [BGW05] construction closely. It uses the $O(\log N)$ way multilinear map to compress the public key of that system from $O(N)$ group elements to $O(\log N)$ elements while keeping the ciphertext overhead and secret key short. We prove static security under a multivariate equivalent of the BGW05] assumption.

The second system uses a general symmetric $O(\log N)$-way multilinear map to similarly compress the public key in [BGW05]. The added flexibility of a symmetric map has both positive and negative consequences. On the negative side, this flexibility allows the adversary to combine extra elements together. To maintain security we must ensure that all user indexes $u \in[N]$ are mapped to integers $\hat{u} \in[O(N \log N)]$ where all $\hat{u}$ have the same Hamming weight. This mapping does not affect ciphertext or private key size. On the positive side, this flexibility allows us to obtain slightly better parameters and base static security on a slightly simpler, though similar, complexity assumption.

The third system is built from a symmetric $O(\log N)$-way map, but we can prove adaptive security of the scheme in generic multilinear groups. This system 
has secret keys of length $O\left(\log ^{3} N\right)$ bits, which is longer than the previous two schemes, but has a tighter security proof in generic groups.

Because the parameters of these systems are logarithmic in $N$, we can let $N$ be exponential, and in particular be as large as the range of a collision resistant hash function (e.g., $N=2^{256}$ ). This, in effect, turns all our broadcast systems into efficient identity-based schemes. A user with identity id $\in\{0,1\}^{*}$ is given the secret key associated with index number $H(\mathrm{id}) \in[N]$ where $H$ is a collision resistant hash whose range is $[N]$. A broadcaster can then transmit to a set of recipients simply by hashing their public identities. For this reason, we describe all our broadcast systems as identity-based broadcast schemes.

Additional related work. Collusion resistant broadcast encryption has been widely studied. Revocation systems (e.g., [NNL01, HS02, GST04, DF02, LSW10) can encrypt to $N-r$ users with ciphertext size of $O(r)$. Further combinatorial solutions (e.g., NP00, DF03]) achieve similar parameters. A broadcast encryption system is said to be recipient-private if broadcast ciphertexts reveal nothing about the intended set of recipients BBW06, LPQ12, FP12. Our broadcast systems are not recipient private, and it is a long-standing open problem to build a low-overhead recipient-private broadcast system. Such a system was recently built using indistinguishability obfuscation [BZ14, but constructing such systems under weaker assumptions remains open.

\section{Preliminaries}

\subsection{Broadcast Encryption}

We begin by defining broadcast encryption. A (public key) identity-based broadcast encryption scheme consists of three randomized algorithms:

Setup $(\mathcal{I D})$ : Sets up a broadcast scheme for identity space $\mathcal{I D}$. It outputs public parameters params as well as a master secret key msk

$\operatorname{KeyGen}(\mathrm{msk}, u)$ : Takes the master secret key and a user $u \in \mathcal{I D}$ and outputs a secret key $\mathrm{sk}_{u}$ for user $u$.

Enc(params, $S)$ : The encryption algorithm takes the public parameters and a polynomial sized set $S \subseteq \mathcal{I D}$ of recipients, and produces a pair ( $\mathrm{Hdr}, K)$. We refer to $\mathrm{Hdr}$ as the header, and $K$ as the message encryption key.

The message is encrypted using a symmetric encryption scheme with the key $K$ to obtain a ciphertext $c$. The overall ciphertext is $(\mathrm{Hdr}, c)$.

Dec(params, $u$, sk $_{u}, S, \mathrm{Hdr}$ ): The decryption algorithm takes the header $\mathrm{Hdr}$ and the secret key for user $u$, and if $u \in S$, outputs the message encryption key $K$. If $u \notin S$, the decryption algorithm outputs $\perp$.

To actually decrypt the overall ciphertext $(\mathrm{Hdr}, c)$, user $u$ runs Dec to obtain $K$, and then decryption $c$ using $K$ to obtain the message.

For correctness, we require that the decryption algorithm always succeeds when it is supposed to. That is, for every (params, msk) output by $\operatorname{Setup}(\mathcal{I D})$, 
every set $S \subseteq \mathcal{I D}$, every sk ${ }_{u}$ output by KeyGen(msk, $u$ ), and (Hdr, $K$ ) outputted by Enc(params, $S)$ where $u \in S$, that $\operatorname{Dec}\left(\right.$ params, $u$, sk $\left._{u}, S, \mathrm{Hdr}\right)=K$.

For security, several notions of security are possible. We start by defining active chosen ciphertext security. For any adversary $\mathcal{A}$, let $\operatorname{EXP}(b)$ denote the following experiment on $\mathcal{A}$ :

Setup: The challenger runs (params, msk $) \leftarrow \operatorname{Setup}(\mathcal{I D})$, and gives $\mathcal{A}$ the public key params.

Secret Key Queries: $\mathcal{A}$ may adaptively make secret key queries for user $u$. In response, the challenger runs $\mathrm{sk}_{u} \leftarrow \operatorname{KeyGen}(\mathrm{msk}, u)$ and gives $\mathrm{sk}_{u}$ to $\mathcal{A}$.

CCA Queries: $\mathcal{A}$ may make chosen ciphertext queries on tuples ( $u, S, \mathrm{Hdr}$ ). The challenger responds with Dec(params, $u, \mathrm{sk}_{u}, S, \mathrm{Hdr}$ ) where $\mathrm{sk}_{u} \leftarrow \operatorname{KeyGen}(\mathrm{msk}, u)$ 1.

Challenge: $\mathcal{A}$ submits a set $S^{*} \subset \mathcal{I D}$, subject to the restriction that $u \notin S^{*}$ for any user $u$ requested in a secret key query. The challenger lets $\left(\mathrm{Hdr}^{*}, K_{0}^{*}\right) \leftarrow$ Enc(params, $\left.S^{*}\right)$. If $b=0$, the challenger gives $\left(\mathrm{Hdr}^{*}, K_{0}^{*}\right)$ to the adversary. If $b=1$, the challenger computes a random key $K_{1}^{*}$ and gives $\left(\mathrm{Hdr}^{*}, K_{1}^{*}\right)$ to the adversary.

More Secret Key Queries: $\mathcal{A}$ may continue making secret key queries for users $u \notin S^{*}$

More CCA Queries: $\mathcal{A}$ may continue making CCA queries on headers $\mathrm{Hdr} \neq$ $\mathrm{Hdr}^{*} 2$.

Guess: $\mathcal{A}$ produces a guess $b^{\prime}$ for $b$.

Using a simple hybrid argument, we can assume the adversary makes only a single challenge query. Let $W_{b}$ be the event that $\mathcal{A}$ outputs 1 in $\operatorname{EXP}(b)$. We define the adaptive $\mathrm{CCA}$ advantage of $\mathcal{A}$, as

$$
\mathrm{BE}^{(\mathrm{adv})}{ }_{\mathcal{A}}=\left|\operatorname{Pr}\left[W_{0}\right]-\operatorname{Pr}\left[W_{1}\right]\right|
$$

Definition 1. A broadcast encryption scheme is adaptively secure under a chosen ciphertext attack (adaptively CCA-secure) if, for all polynomial time adver-

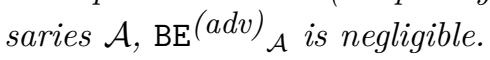

We will also consider several weaker notions of security. For example, we get static security if we require $\mathcal{A}$ to commit to the challenge set $S^{*}$ before seeing the public parameters. We also get CPA security if we do not allow chosen ciphertext queries. In this paper, we will be focusing on the following notion of static CPA security, but will also discuss the other variants:

Definition 2. A broadcast encryption scheme is statically secure under a chosen plaintext attack (statically CPA-secure) if, for all polynomial time adversaries $\mathcal{A}$ that must commit to $S^{*}$ before seeing the public parameters and cannot make $C C A$ queries, $\mathrm{BE}^{(a d v)} \mathcal{A}$ is negligible.

\footnotetext{
${ }^{1}$ Another variation is to have the challenger maintain a table of $\left(u, \mathbf{s k}_{u}\right)$ pairs, and only run KeyGen once for a particular user, using a single $\mathbf{s k}_{u}$ to answer multiple secret key and CCA queries. Note that the correctness of a broadcast scheme implies that this does not affect CCA queries.

2 Another potentially stronger variation is to require $(S, \mathrm{Hdr}) \neq\left(S^{*}, \mathrm{Hdr}^{*}\right)$
} 


\subsection{Multilinear Maps}

We now review multilinear maps BS03, GGH13a, CLT13. A multilinear map consists of two algorithms:

Setup $(n)$ : Sets up an $n$-linear map. It outputs $n$ groups $\mathbb{G}_{1}, \ldots, \mathbb{G}_{n}$ of prime order $p$, along with generators $g_{i} \in \mathbb{G}_{i}$. We call $\mathbb{G}_{1}$ the source group, $\mathbb{G}_{n}$ the target group, and $\mathbb{G}_{2}, \ldots, \mathbb{G}_{n-1}$ intermediate groups.

$e_{i, j}(g, h)$ : Takes in two elements $g \in \mathbb{G}_{i}$ and $h \in \mathbb{G}_{j}$ with $i+j \leq n$, and outputs an element of $\mathbb{G}_{i+j}$ such that

$$
e_{i, j}\left(g_{i}^{a}, g_{j}^{b}\right)=g_{i+j}^{a b}
$$

We often omit the subscripts and just write $e$. We can also generalize $e$ to multiple inputs as $e\left(h^{(1)}, \ldots, h^{(k)}\right)=e\left(h^{(1)}, e\left(h^{(2)}, \ldots, h^{(k)}\right)\right)$.

We sometimes call $g_{i}^{a}$ as a level- $i$ encoding of $a$. The scalar $a$ itself could be referred to as a level-0 encoding of $a$. Then the map $e$ combines a level $i$ encoding and a level $j$ encoding, and produces a level $i+j$ encoding of the product.

We will make use of asymmetric multilinear maps. In such maps, groups are indexed by integer vectors rather than integers. The pairing operations maps $\mathbb{G}_{\mathbf{v}_{1}} \times \mathbb{G}_{\mathbf{v}_{2}}$ into $\mathbb{G}_{\mathbf{v}_{1}+\mathbf{v}_{2}}$. More precisely, we have the following algorithms:

$\operatorname{Setup}(\mathbf{n})$ Sets up an $\mathbf{n}$-linear map, where $\mathbf{n} \in \mathbb{Z}^{\ell}$ is some positive integer vector. It outputs a description of groups $\mathbb{G}_{\mathbf{v}}$ of prime order $p$ where $\mathbf{v}$ are non-negative integer vectors and $\mathbf{v} \leq \mathbf{n}$ (that is, the comparison must hold component-wise). It also outputs a description of generators $g_{\mathbf{v}} \in \mathbb{G}_{\mathbf{v}} 3$. Let $\mathbf{e}_{i}$ be the $i$ th standard basis vector, with a 1 at position $i$ and a 0 elsewhere. We call $\mathbb{G}_{\mathbf{e}_{i}}$ the $i$ th source group, $\mathbb{G}_{\mathbf{n}}$ the target group, and the rest of the $\mathbb{G}_{\mathbf{v}}$ groups are intermediate groups.

$e_{\mathbf{v}_{1}, \mathbf{v}_{2}}(g, h)$ Takes in two elements $g \in \mathbb{G}_{\mathbf{v}_{1}}$ and $h \in \mathbb{G}_{\mathbf{v}_{2}}$ with $\mathbf{v}_{1}+\mathbf{v}_{2} \leq \mathbf{n}$, and outputs an element of $\mathbb{G}_{\mathbf{v}_{1}+\mathbf{v}_{2}}$ such that

$$
e_{\mathbf{v}_{1}, \mathbf{v}_{2}}\left(g_{\mathbf{v}_{1}}^{a}, g_{\mathbf{v}_{2}}^{b}\right)=g_{\mathbf{v}_{1}+\mathbf{v}_{2}}^{a b}
$$

We often omit the subscripts and just write $e$. We can also generalize $e$ to multiple inputs as $e\left(h^{(1)}, \ldots, h^{(k)}\right)=e\left(h^{(1)}, e\left(h^{(2)}, \ldots, h^{(k)}\right)\right)$.

We note that current candidates of multilinear maps GGH13a, CLT13] depart from the ideal notions of multilinear maps described above. In particular, in these candidates, representations of group elements are not unique and contain a noise term that can cause errors during group and multilinear operations. While we present our constructions using ideal multilinear maps for simplicity, we stress that our constructions can easily be instantiated using current nonideal candidates. We need multilinear maps with the following properties:

${ }^{3}$ There may be an exponential number of groups and generators. The setup algorithm outputs a set of parameters from which the groups $\mathbb{G}_{\mathbf{v}}$ and generators $g_{\mathbf{v}}$ can be derived. In particular, each $g_{\mathbf{v}}$ can be derived from the pairing operation and $\left\{g_{\mathbf{e}_{i}}\right\}$, where $\mathbf{e}_{i}$ is the $i$ th standard basis vector 
- A way to hide the group and multilinear operations that lead to a particular element. In current multilinear maps, this is obtained by performing a re-randomization procedure which makes the representation of an element statistically independent of the operations that lead to that element.

- A way, given any representation of an element in the target group, to "extract" a canonical representation of that element. This is handled by a "zerotest parameter" in current maps.

- The ability for the person who sets up the map to compute elements of the form $g^{\alpha^{x}}$ for exponentially-large $x$. In ideal multilinear maps, this would be accomplished by computing $z=\alpha^{x}$ in $\mathbb{Z}_{p}$, and then computing $g^{z}$. However, with current multilinear maps, it is not possible for normal users to compute $g^{z}$ for a specific $z$ of their choice 4 . However, the person who sets up the map knows a trapdoor that does allow computing $g^{z}$ for any $z \in \mathbb{Z}_{p}$.

- The ability to generate asymmetric multilinear maps for any positive integer vector $\mathbf{n} \in \mathbb{Z}^{\ell}$. Section 4.3 of [GGH13a] shows how to do this.

- A way to make sure the noise growth does not cause any errors during normal execution of our protocols. Since there is no circular dependence between the parameters of the multilinear map and the number of operations our protocols require, we can set the parameters so the noise stays small enough to avoid errors.

\section{Our Asymmetric Multilinear Map Construction}

In this section, we give our first construction of identity-based broadcast encryption from multilinear maps. Our starting point is the scheme of Boneh, Gentry, and Waters [BGW05], henceforth referred to as the BGW scheme. Recall in their scheme, the public parameters consist of $O(N)$ source group elements (where $N$ is the number of users), secret keys and headers are a constant number of source group elements, and the message encryption key is a group element in the target group. Our goal is to shrink the public key size to $O(\log N)$ group elements. We accomplish this by embedding the BGW scheme in a multilinear map, where the BGW parameters lie in an intermediate group. The BGW public parameters can then be derived from a small number of elements in the source group of the map - these few source group elements are the new public key.

In more detail, the significant component of the BGW public key are the elements $Z_{1}=g_{1}^{\alpha}, Z_{2}=g_{1}^{\alpha^{2}}, \ldots, Z_{N}=g_{1}^{\alpha^{N}}, Z_{N+2}=g_{1}^{\alpha^{N+2}}, \ldots, Z_{2 N}=g_{1}^{\alpha^{2 N}}$. The rest of the BGW public keys, secret keys, and header components are also element in $\mathbb{G}_{1}$, whereas the message encryption key is an element in the group $\mathbb{G}_{2}$.

Let $N=2^{n}-1$ for some integer $n$, and let $\mathbf{n}=(\overbrace{1, \ldots, 1}^{n+1})$ be the vector of $n+1$ s. Our idea is to use an asymmetric multilinear map, where the target group is $\mathbb{G}_{2 \mathbf{n}}$. We note that pairing two elements in $\mathbb{G}_{\mathbf{n}}$ gives an element in $\mathbb{G}_{2 \mathbf{n}}$. Thus, while the entire multilinear map is asymmetric, the pairing operation acts symmetrically on the group $\mathbb{G}_{\mathbf{n}}$. Now we replace the groups $\mathbb{G}_{1}$ and $\mathbb{G}_{2}$ in the

\footnotetext{
${ }^{4}$ Instead, users can compute $g^{z}$ for a random, but unknown, $z$.
} 
BGW scheme with $\mathbb{G}_{\mathbf{n}}$ and $\mathbb{G}_{2 \mathbf{n}}$. Thus $Z_{u}=g_{\mathbf{n}}^{\alpha^{u}}$. Rather than explicitly include the $Z_{u}$ in the public parameters, we give a few group elements in the groups $\mathbb{G}_{\mathbf{e}_{i}}$ where $\mathbf{e}_{i}$ are the standard basis vectors. Specifically, we provide the parameters $X_{i}=g_{\mathbf{e}_{i}}^{\alpha^{\left(2^{i}\right)}}$ for $i=0, \ldots, n-1$. By pairing various subsets of these $X_{i}$ together, we can build all of the $Z_{u}$ for $u \leq 2^{n}-1=N$. In particular, if $u=\sum_{i=0}^{n-1} u_{i} 2^{i}$ is the binary representation of $u$, then

$$
Z_{u}=e\left(X_{0}^{u_{0}} g_{\mathbf{e}_{0}}^{1-u_{0}}, X_{1}^{u_{1}} g_{\mathbf{e}_{1}}^{1-u_{1}}, \ldots, X_{n-1}^{u_{n-1}} g_{\mathbf{e}_{n-1}}^{1-u_{n-1}}, g_{\mathbf{e}_{n}}\right)
$$

where $X_{i}^{0} g_{\mathbf{e}_{i}}^{1}=g_{\mathbf{e}_{i}}$ and $X_{i}^{1} g_{\mathbf{e}_{i}}^{0}=X_{i}$

To allow computation of $Z_{u}$ for $u \geq 2^{n}+1=N+2$, we might decide to publish $g_{\mathbf{e}_{n}}^{\alpha^{\left(2^{n}\right)}}$. However, this would allow computation of $Z_{N+1}$, which will break the security of the BGW scheme. Therefore, we instead publish $X_{n}=g_{\mathbf{e}_{n}}^{\alpha^{\left(2^{n}+1\right)}}$. Then, for $u \in[N+2,2 N]$, let $u^{\prime}=u-\left(2^{n}+1\right)=\sum_{i=0}^{n-1} u_{i}^{\prime} 2^{i}$. Then we can write

$$
Z_{u}=e\left(X_{0}^{u_{0}^{\prime}} g_{\mathbf{e}_{0}}^{1-u_{0}^{\prime}}, X_{1}^{u_{1}^{\prime}} g_{\mathbf{e}_{1}}^{1-u_{1}^{\prime}}, \ldots, X_{n-1}^{u_{n-1}^{\prime}} g_{\mathbf{e}_{n-1}}^{1-u_{n-1}^{\prime}}, X_{n}\right)
$$

Now we make the observation that $O(\log N)$ graded encodings remain efficient even up to exponential $N$. Therefore, we can actually make our scheme identitybased, where identities are bit strings of length $n$ with the caveat that the $0^{n}$ is not a valid identity. Now we give our entire construction:

Construction 1. Let Setup' be the setup algorithm for a multilinear map, where groups have order $p$. Our first identity-based broadcast scheme consists of the following algorithms:

Setup $(n)$ : Takes as input the length $n$ of identities. Let $\mathcal{I D}=\{0,1\}^{n} \backslash\left\{0^{n}\right\}$ be the identity space. Let $\mathbf{n}$ be the all-ones vector of length $n+1$. Run Setup' on $2 \mathbf{n}$, obtaining the public parameters params' for a multilinear map with target group $\mathbb{G}_{2 \mathbf{n}}$.

Choose a random $\alpha \in \mathbb{Z}_{p}$ and let $X_{i}=g_{\mathbf{e}_{i}}^{\alpha^{\left(2^{i}\right)}}$ for $i=0, \ldots, n-1$ and let $X_{n}=g_{\mathbf{e}_{n}}^{\alpha^{\left(2^{n}+1\right)}}$. Also choose a random $\gamma \in \mathbb{Z}_{p}$ and let $Y=g_{\mathbf{n}}^{\gamma}$. Lastly, let $W=g_{2 \mathbf{n}}^{\alpha^{\left(2^{n}\right)}}$. The public key is

$$
\text { params }=\left(\text { params }^{\prime}, W,\left\{X_{i}\right\}_{i \in\{0, \ldots, n\}}, Y\right)
$$

The master secret key is $(\alpha, \gamma)$.

KeyGen(params, $\alpha, \gamma, u)$ : The secret key for identity $u \in\left[1,2^{n}-1\right]$ is $\mathrm{sk}_{u}=g_{\mathbf{n}}^{\gamma \alpha^{u}}$. Enc(params, $S)$ : Recall that we can compute $Z_{j}$ for $j \in\left[1,2^{n}-1\right]$ from the public parameters $\left\{X_{i}\right\}_{i \in\{0, \ldots, n-1\}}$. Pick a random $t \in \mathbb{Z}_{p}$ and compute the key and header as

$$
\begin{aligned}
K & =W^{t}=g_{2 \mathbf{n}}^{t \alpha^{\left(2^{n}\right)}} \text { and } \\
\mathrm{Hdr} & =\left(g_{\mathbf{n}}^{t},\left(Y \cdot \prod_{u \in S} Z_{2^{n}-u}\right)^{t}\right)=\left(g_{\mathbf{n}}^{t}, g_{\mathbf{n}}^{t\left(\gamma+\sum_{u \in S} \alpha^{\left(2^{n}-u\right)}\right)}\right)
\end{aligned}
$$


$\operatorname{Dec}\left(\right.$ params, $\left.u, \mathrm{sk}_{u}, S, \mathrm{Hdr}\right):$ If $u \notin S$, output $\perp$. Otherwise, write $\mathrm{Hdr}$ as $\left(C_{0}, C_{1}\right)$. Recall that we can compute $Z_{j}$ for $j \in\left[2^{n}+1,2^{n+1}\right]$. Output

$$
K=\frac{e\left(Z_{u}, C_{1}\right)}{e\left(\left(\mathrm{sk}_{u} \cdot \prod_{j \in S, j \neq u} Z_{2^{n}-j+u}\right), C_{0}\right)}
$$

If $C_{0}$ and $C_{1}$ are as above, then we can write $K=g_{2 \mathbf{n}}^{c}$ where

$$
c=\alpha^{u} t\left(\gamma+\sum_{j \in S} \alpha^{\left(2^{n}-j\right)}\right)-\left(\gamma \alpha^{u}+\sum_{j \in S, j \neq u} \alpha^{\left(2^{n}-j+u\right)}\right) t
$$

Most of the terms cancel, leaving $c=t \alpha^{2^{n}}$ as desired.

Implementation details. As mentioned in Section2 2 there are some minor complications with implementing our scheme using current multilinear map constructions GGH13a, CLT13, but we stress that these complications do not affect the semantics of our scheme. First, during normal operations, computing $g_{1}^{\alpha}$ for a random $\alpha$ involves computing a "level-0" encoding of a random (unknown) $\alpha$, and then pairing with $g_{1}$. In order to compute $g_{1}^{\alpha^{2}}$, we would pair $g_{1}$ with the level-0 encoding twice. However, the noise growth with repeated pairing operations would prevent us from computing $g_{1}^{\alpha^{\left(2^{i}\right)}}$ for sufficiently high powers of $i$. Instead, the setup algorithm must choose an explicit (known) $\alpha \in \mathbb{Z}_{p}$, compute the various $\alpha^{2^{i}}$, encode these powers as level-0 encodings, and only then pair with $g_{1}$. This requires knowing the secrets used to set up the multilinear map, meaning the broadcaster must set up the map himself and cannot rely on maps generated by trusted parties. Note, however, that this exponentiation is only required during setup, and not encryption or decryption, meaning the secrets can be discarded immediately after setup, and anyone can still broadcast using the public parameters.

To make sure the header does not leak any important information, we also need to re-randomize the header components. This means re-randomization parameters need to be included for the group $\mathbb{G}_{\mathbf{n}}$. No other re-randomization parameters are necessary.

Before discussing security, we must discuss our new security assumption, which is closely related to the bilinear Diffe-Hellman Exponent assumption (BDHE) as used in BGW.

\subsection{The Hybrid Diffie-Hellman Exponent Assumption (HDHE) Assumption}

We define the (computational) $n$-Hybrid Diffie-Hellman Exponent problem as follows: Let params' $\leftarrow$ Setup $^{\prime}(2 \mathbf{n})$ where $\mathbf{n}$ is the all-ones vector of length $n+1$. Choose $\alpha \in \mathbb{Z}_{p}$ at random, and let $X_{i}=g_{\mathbf{e}_{i}}^{\alpha^{\left(2^{i}\right)}}$ for $i=0, \ldots, n-1$ and 
$X_{n}=g_{\mathbf{e}_{n}}^{\alpha^{\left(2^{n}+1\right)}}$. Choose a random $t \in \mathbb{Z}_{p}$ and let $V=g_{\mathbf{n}}^{t}$. Given $\left(\left\{X_{i}\right\}_{i \in\{0, \ldots, n-1\}}\right.$, $V)$, the goal is to compute $K=g_{2 \mathbf{n}}^{t \alpha^{\left(2^{n}\right)}}$.

We now define the decisional $n$-Hybrid Diffie-Hellman Expoent problem as, given the tuple $\left(\left\{X_{i}\right\}_{i \in\{0, \ldots, n-1\}}, V, K\right)$ where $K$ is either $g_{2 \mathbf{n}}^{t \alpha^{\left(2^{n}\right)}}$ or a random element of $\mathbb{G}_{2 \mathbf{n}}$, to distinguish the two cases.

Definition 3. We say the decisional n-Hybrid Diffie-Hellman Exponent assumption holds for Setup' if, for any polynomial $n$ and probabilistic polynomial time algorithm $\mathcal{A}, \mathcal{A}$ has negligible advantage in solving the $n$-Hybrid Diffie-Hellman Exponent problem.

Given the $X_{i}$ for $i=0, \ldots, n-1$, it is straightforward to compute $g_{\mathbf{n}}^{\alpha^{j}}$ for any $j \in\left[0,2^{n}-1\right]$. Moreover, including $X_{n}$, it is straightforward to extend this to $j \in\left[2^{n}+1,2^{n+1}\right]$. However, computing $K=g_{2 \mathbf{n}}^{t \alpha^{\left(2^{n}\right)}}$ from the $X_{i}$ and $V$ appears difficult. The reason is that we only have one term that depends on $t$, namely $V$. So to compute $K$, we would need to pair $V$ with some combination of the $X_{i}$. In other words, we need to be able to compute $g_{\mathbf{n}}^{\alpha^{\left(2^{n}\right)}}$ from the $X_{i}$. However, since $\mathbf{n}$ has a one in each component, we can never pair any of the $X_{i}$ with itself. This means we can only compute products of terms of the form $e\left(X_{0}^{s_{0}}, X_{1}^{s_{1}}, \ldots, X_{n}^{s_{n}}\right)$ for $s_{i} \in\{0,1\}$, where we take $X_{i}^{0}=g_{\mathbf{e}_{1}}$. Notice that we can never include an $X_{n}$, since then we would already exceed the desired degree of $2^{n}$. Put another way, we can only compute products of terms of the form

$$
\prod_{\mathbf{n}} \prod_{i \in S} \alpha^{\left(2^{i}\right)}
$$

where $S \subseteq[0, n-1]$. However, $\prod_{i \in S} \alpha^{2^{i}}=\alpha^{\sum_{i \in S} 2^{i}}$, and $\sum_{i \in S} 2^{i}<2^{n}$ for all subsets $S \subseteq[0, n-1]$. This is the basis for our assumption that the $n$-HDHE assumption is hard. In the full version BWZ14, we discuss the difficulty of our assumption in the generic multilinear map model.

\subsection{Security of Our Construction}

With our assumption defined, we can now state and prove the security of our scheme:

Theorem 2. Let Setup' be the setup algorithm for a multilinear map, and suppose that the decisional n-Hybrid Diffie-Hellman Exponent assumption holds for Setup'. Then the scheme in Construction 1 is a statically secure identity-based broadcast encryption scheme.

Proof. Our proof closely follows the security proof for BGW BGW05. Suppose we have an adversary $\mathcal{A}$ that breaks the security of the scheme. We use $\mathcal{A}$ to build an adversary $\mathcal{B}$ that breaks the decisional $n$-HDHE problem for Setup'. $\mathcal{B}$ works as follows: 
- $\mathcal{B}$ obtains a challenge tuple (params' $\left.,\left\{X_{i}\right\}_{i \in[0, n]}, V, K\right)$ where:

- params $^{\prime} \leftarrow \operatorname{Setup}^{\prime}(2 \mathbf{n})$ where $\mathbf{n}$ is the all-ones vector of length $n+1$.

- $X_{i}=g_{\mathbf{e}_{i}}^{\alpha^{2^{i}}}$ for $i=0, \ldots, n-1$ for a random $\alpha \in \mathbb{Z}_{p}$.

- $X_{n}=g_{\mathbf{e}_{n}}^{\alpha^{2^{n}+1}}$

- $V=g_{\mathbf{n}}^{t}$ for a random $t \in \mathbb{Z}_{p}$

- $K=g_{2 \mathbf{n}}^{t 2^{2^{n}}}$ or $K$ is a random group element in $\mathbb{G}_{2 \mathbf{n}}$.

- $\mathcal{B}$ simulates $\mathcal{A}$ until $\mathcal{A}$ submits a subset $S \subseteq\left[1,2^{n}-1\right]$ of users that $\mathcal{A}$ will challenge.

- $\mathcal{B}$ chooses a random $r \in \mathbb{Z}_{p}$. It sets

$$
Y=\frac{g_{\mathbf{n}}^{r}}{\prod_{u \in S} Z_{2^{n}-u}}
$$

where the $Z_{j}$ are calculated from the $X_{i}$ as before. This amounts to setting

$$
\gamma=r-\sum_{u \in S} \alpha^{2^{n}-u}
$$

Since $r$ is uniform in $\mathbb{Z}_{p}$ and independent of $\alpha$, so is $\gamma . \mathcal{B}$ also computes

$$
W^{\prime}=e\left(g_{\mathbf{e}_{0}}, g_{\mathbf{e}_{1}}, \ldots, g_{\mathbf{e}_{n-2}}, X_{n-1}, g_{\mathbf{e}_{n}}\right)
$$

and

$$
W=e\left(W^{\prime}, W^{\prime}\right)
$$

Observe that $W=g_{2 \mathbf{n}}^{\alpha^{2^{n}}}$.

- $\mathcal{B}$ gives $\mathcal{A}$ the public parameters $\left(W,\left\{X_{i}\right\}_{i \in[0, n]}, Y\right)$

- Now $\mathcal{A}$ is allowed to ask for private keys for users $u \notin S$. $\mathcal{B}$ computes

$$
\mathrm{sk}_{u}=\frac{Z_{u}^{r}}{\prod_{j \in S} Z_{2^{n}-j+u}}
$$

Observe that

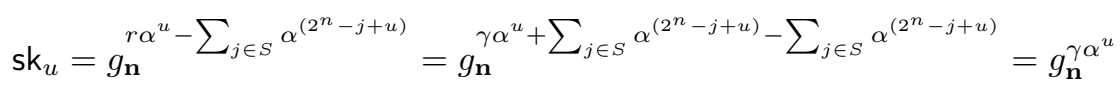

as desired.

- When $\mathcal{A}$ asks for the challenge, $\mathcal{B}$ lets $\mathrm{Hdr}=\left(V, V^{r}\right)$ and responds with $(\mathrm{Hdr}, K)$. Observe that

$$
V^{r}=g_{\mathbf{n}}^{r t}=g_{\mathbf{n}}^{t\left(\gamma+\sum_{u \in S} \alpha^{\left(2^{n}-u\right)}\right)}
$$

which means $\left(V, V^{r}\right)$ is a valid header for the set $S$. Also observe that if $K=g_{2 \mathbf{n}}^{t \alpha^{\left(2^{n}\right)}}$, then $K$ is the correct key for this header.

- When $\mathcal{A}$ returns a guess $b$ for which $K$ it is given, $\mathcal{B}$ returns $b$ as its guess.

As shown above, $\mathcal{B}$ perfectly simulates the view of $\mathcal{A}$ in the broadcast encryption security game. Therefore, $\mathcal{B}$ has the same advantage as $\mathcal{A}$, which must therefore be negligible, as desired. 


\section{Our Symmetric Multilinear Map Construction}

In this section, we give our second construction of broadcast encryption, this time from traditional symmetric multilinear maps. That is, we do not require the more complicated asymmetric structure of Construction 1, but can use a basic multilinear map. The idea, however, is very similar. We implement BGW BGW05] in middle levels of the multilinear map, and use elements in the bottom level to generate the BGW public parameters. Similar to the graded encoding scheme, the BGW parameters will have the form $Z_{u}=g_{n}^{\alpha^{u}}$, which can be computed from the public parameters $X_{i}=g_{1}^{\alpha^{\left(2^{i}\right)}}$.

However, we run into a problem. With asymmetric maps, we could enforce that $X_{i}$ could not be paired itself. This was used to ensure that $Z_{2^{n}}$ was not computable given $X_{i}$ for $i=0, \ldots, n$. However, in the symmetric multilinear map setting, $X_{n-1}$ could be paired with itself, giving $Z_{2^{n}}$. Instead, we create a hole by limiting the total number of $X_{i}$ that can be paired together. If we allow only $n-1$ of them to be paired together, the first hole occurs at $Z_{2^{n}-1}$. We therefore set $N=2^{n}-2$ so that the hole is at $N+1$ as in BGW.

Notice that a second hole occurs at $Z_{2^{n}+2^{n-1}-1}$, and since $2^{n}+2^{n-1}-1<$ $2\left(2^{n}-2\right)=2 N$, we can not yet compute all the $Z_{u}$ needed by BGW. One possible fix is to include extra $X_{i}$ that can be used to fill in the unwanted holes. Instead, we opt to restrict the bit representations of all identities in the system to having the same Hamming weight. We show that this allows the computation of all the necessary $Z_{u}$.

We now describe our scheme:

Construction 3. Let Setup' be the setup algorithm for a multilinear map, where groups have order p. Our second identity-based broadcast scheme consists of the following algorithms:

$\operatorname{Setup}(n, \ell)$ Sets up a broadcast scheme for $n$-bit identities with Hamming weight $\ell$. Run Setup' on $n+\ell-1$, obtaining the public parameters params' for a multilinear map with target group $\mathbb{G}_{n+\ell-1}$. Let $\alpha, \gamma \in \mathbb{Z}_{p}$ be chosen at random. Let $W=g_{n+\ell-1}^{\alpha^{\left(2^{n}-1\right)}}$. Compute $X_{i}=g_{1}^{\alpha^{\left(2^{i}\right)}}$ for $i=0, \ldots, n$. Lastly, let $Y=g_{n-1}^{\gamma}$. Output

$$
\text { params }=\left(\text { params }^{\prime}, W,\left\{X_{i}\right\}_{i \in[0, n]}, Y\right)
$$

KeyGen(params, $\alpha, \gamma, u)$ The secret key for an identity $u \in\{0,1\}^{n}$ of Hamming weight $\ell$ is

$$
\mathrm{sk}_{u}=g_{n-1}^{\gamma \alpha^{u}}
$$

Enc(params, $S)$ Let $Z_{j}=g_{n-1}^{\alpha^{j}}$. We will show shortly that we can compute all of the necessary $Z_{j}$ from the $X_{i}$. Pick a random $t \in \mathbb{Z}_{p}$ and compute the key and header as

$$
\begin{aligned}
K & =W^{t}=g_{n+\ell-1}^{t \alpha^{\left(2^{n}-1\right)}} \text { and } \\
\operatorname{Hdr} & =\left(g_{\ell}^{t},\left(Y \prod_{u \in S} Z_{2^{n}-1-u}\right)^{t}\right)=\left(g_{\ell}^{t}, g_{n-1}^{t\left(\gamma+\sum_{u \in S} \alpha^{\left(2^{n}-1-u\right)}\right)}\right)
\end{aligned}
$$


$\operatorname{Dec}\left(\right.$ params, $\left.u, \mathrm{sk}_{u}, S, \mathrm{Hdr}\right)$ If $u \notin S$, output $\perp$. Otherwise, write $\mathrm{Hdr}=\left(C_{0}, C_{1}\right)$. Also let $Z_{u}^{\prime}=g_{\ell}^{\alpha^{u}}$. We will shortly show that $Z_{u}^{\prime}$ can be computed from the $X_{i}$. Compute

$$
K=\frac{e\left(Z_{u}^{\prime}, C_{1}\right)}{e\left(\mathrm{sk}_{u} \cdot \prod_{j \in S, j \neq u} Z_{2^{n}-1-j+u}, C_{0}\right)}
$$

If $C_{0}, C_{1}$ are as above, notice that we can write $K=g_{n+\ell-1}^{c}$ where

$$
c=\alpha^{u} t\left(\gamma+\sum_{j \in S} \alpha^{2^{n}-1-j}\right)-\left(\gamma \alpha^{u}+\sum_{j \in S, j \neq u} \alpha^{2^{n}-1-j+u}\right) t=t \alpha^{2^{n}-1}
$$

as desired.

We need to show how to compute $Z_{j}$ and $Z_{j}^{\prime}$.

Claim. Let $Z_{j}=g_{n-1}^{\alpha^{j}}$ and $Z_{j}^{\prime}=g_{\ell}^{\alpha^{j}}$. Let $X_{i}=g_{1}^{\alpha^{\left(2^{i}\right)}}$ for $i=0, \ldots, n$. Then, using group multiplications and paring operations on the $X_{i}$, it is possible to compute:

- $Z_{j}^{\prime}$ for $j \in\left[1,2^{n}-2\right]$ of weight exactly $\ell$.

$-Z_{2^{n}-1-j}$ for $j \in\left[1,2^{n}-2\right]$ of weight exactly $\ell$.

$-Z_{2^{n}-1-j+u}$ for $j, u \in\left[1,2^{n}-2\right], j \neq u$ of weight exactly $\ell$.

Proof. Let $h(j)$ denote the Hamming weight of $j$. First, observe that we can easily compute $g_{h(j)}^{\alpha^{j}}$ for $j \in\left[2^{n}-1\right]$ by paring together $X_{i}$ where the $i$ th bit of $j$ is 1 . This allows us to compute the $Z_{j}^{\prime}$. We can also compute $g_{n-\ell}^{\alpha^{\left(2^{n}-1-j\right)}}$ for any $j$ of weight exactly $\ell$. Thus, we can pair with $g_{\ell-1}$ to get $Z_{2^{n}-1-j}$.

Now we show how to compute $Z_{2^{n}-1-j+u} \cdot 2^{n}-1-j$, written as a bit string, has Hamming weight $n-\ell$. Therefore, write $2^{n}-1-j=\sum_{i \in T} 2^{i}$ for some subset $T \subseteq[0, n-1]$ of size $n-\ell$. Similarly, write $u=\sum_{i \in U} 2^{i}$ for some subset $U \subseteq[0, n-1]$ of size $\ell$. Notice that $U$ and $T$ are only disjoint if $2^{n}-1-j+u=$ $2^{n}-1$, in which case $j=u$. Since we do not allow this case, there must be some $\hat{i} \in[0, n-1]$ inside $U$ and $T$. Then we can write

$$
2^{n}-1-j+u=\left(\sum_{i \in T \backslash\{\hat{i}\}} 2^{i}\right)+\left(\sum_{i \in U \backslash\{\hat{i}\}} 2^{i}\right)+2^{\hat{i}+1}
$$

which is the sum of $n+\ell-1$ powers of two. This means we can write

$$
Z_{2^{n}-1-j+u}=e\left(\left\{X_{i}\right\}_{i \in T \backslash\{\hat{i}\}},\left\{X_{i}\right\}_{i \in U \backslash\{\hat{i}\}}, X_{\hat{i}+1}\right)
$$

which is the pairing of $n+\ell-1$ of the $X_{i}$, as desired. 
Setting $n$ and $\ell$. Suppose we want to handle $\lambda$-bit identities. We would map those identities to bit strings of length $n$ and weight $\ell$. Therefore, we need

$$
\lambda \geq \log _{2}\left(\begin{array}{l}
n \\
\ell
\end{array}\right)
$$

A simple solution which minimizes $n$ (and hence the number of elements in the public parameters $)$ is to set $n=\lambda+\left\lceil\left(\log _{2} \lambda\right) / 2\right\rceil+1$ and $\ell=\lfloor n / 2\rfloor$. However, for existing multilinear map constructions, the multilinearity itself is expensive, so we might try to minimize the total multilinearity $n+\ell-1$. When $\ell=\lfloor n / 2\rfloor$, the total multilinear is roughly $1.5\left(\lambda+\left(\log _{2} \lambda\right) / 2\right)$. However, setting $n \approx 1.042\left(\lambda+\left(\log _{2} \lambda\right) / 2\right)$ and $\ell \approx 0.398(\lambda+(\log \lambda) / 2)$ gives us roughly $2^{\lambda}$ identities with total multilinearity about $1.440(\lambda+(\log \lambda) / 2)$, slightly beating the trivial construction. The following table gives the settings of $n$ and $\ell$ which minimize the total multilinearity for common identity lengths:

\begin{tabular}{|c|c|c|c|}
\hline Length of identities $(\lambda)$ & $n$ & $\ell$ & Total Multilinearity $(n+\ell-1)$ \\
\hline 128 & 138 & 52 & 189 \\
\hline 160 & 175 & 62 & 236 \\
\hline 256 & 272 & 103 & 374 \\
\hline 512 & 545 & 200 & 744 \\
\hline
\end{tabular}

Implementation. As with Construction 1, we must take advantage of the secrets used to construct the multilinear map to compute the $X_{i}$. We also need to rerandomize the header components. This time, however, there are two groups that need re-randomization terms: $\mathbb{G}_{\ell}$ and $\mathbb{G}_{n-1}$. No other re-randomization parameters are necessary.

\subsection{The Multilinear Diffie-Hellman Exponent Assumption}

We define the computational $(n, \ell)$-multilinear Diffie-Hellman Exponent $((n, \ell)$ MDHE) Problem as follows: Let params $\leftarrow \operatorname{Setup}^{\prime}(n+\ell-1)$. Choose random $\alpha, t \in \mathbb{Z}_{p}$, and let $X_{i}=g_{1}^{\alpha^{\left(2^{i}\right)}}$ for $i=0, \ldots, n$. Let $V=g_{\ell}^{t}$. Given $\left(\left\{X_{i}\right\}_{i \in[0, n]}, V\right)$, the goal is to compute $K=g_{n+\ell-1}^{t \alpha^{\left(2^{n}-1\right)}}$.

As before, we define the decisional version as the problem of distinguishing $K$ from a random element in $G_{n+\ell-1}$.

Definition 4. We say the decisional $(n, \ell)$-multilinear Diffie-Hellman Exponent assumption holds for Setup' if, for any polynomial $n$ and probabilistic polynomial time algorithm $\mathcal{A}, \mathcal{A}$ has negligible advantage in solving the $(n, \ell)$-multilinear Diffie-Hellman Exponent problem.

This problem appears difficult for the same reasons as the $n$-HDHE assumption from Section [3. Computing $K=g_{n+\ell-1}^{t \alpha^{\left(2^{n}-1\right)}}$ requires pairing $V=g_{\ell}^{t}$ with a term $g_{n-1}^{\alpha^{\left(2^{n}-1\right)}}$, which must in turn be computed from the $X_{i}$. However, there is no way to pair at most $n-1$ of the $X_{i}$ to create the desired exponent $2^{n}-1$. In the full version BWZ14, we discuss the difficulty of the $(n, \ell)$-MDHE problem in the generic multilinear map model. 


\subsection{Security of Our Construction}

With our assumption defined, we can now state the security of our scheme:

Theorem 4. Let Setup' be the setup algorithm for a multilinear, and suppose that the decisional $(n, \ell)$-multilinear Diffie-Hellman Exponent assumption holds for Setup'. Then the scheme in Construction 3 is a secure identity-based broadcast encryption scheme.

Proof. Again, our proof follows BGW [BGW05]. Suppose we have an adversary $\mathcal{A}$ that breaks the security of the scheme. We use $\mathcal{A}$ to build an adversary $\mathcal{B}$ that breaks the decisional MDHE problem for Setup'. $\mathcal{B}$ works as follows:

- $\mathcal{B}$ obtains a challenge tuple (params', $\left\{X_{i}\right\}_{i \in[0, n+1]}, V, K$ ) where:

- params $^{\prime} \leftarrow \operatorname{Setup}^{\prime}(n+\ell-1)$

- $X_{i}=g_{1}^{\alpha^{\left(2^{i}\right)}}$ for $i=0, \ldots, n$ for a random $\alpha \in \mathbb{Z}_{p}$

- $V=g_{\ell}^{t}$ for a random $t \in \mathbb{Z}_{p}$

- $K=g_{n+\ell-1}^{\left.t \alpha^{\left(2^{n}\right.}-1\right)}$ or $K$ is a random element in $\mathbb{G}_{n+\ell-1}$.

- $\mathcal{B}$ simulates $\mathcal{A}$ until $\mathcal{A}$ submits a subset $S \subseteq\left[1,2^{n}-2\right]$ of users that all have Hamming weight $\ell$.

- $\mathcal{B}$ chooses a random $r \in \mathbb{Z}_{p}$. It sets

$$
Y=g_{n-1}^{r} / \prod_{u \in S} Z_{2^{n}-1-s}
$$

where the $Z_{j}$ are calculated from the $X_{i}$ as before. This amounts to setting

$$
\gamma=r-\sum_{u \in S} \alpha^{2^{n}-1-u}
$$

Since $r$ is uniform in $\mathbb{Z}_{p}$ and independent of $\alpha$, so is $\gamma . \mathcal{B}$ computes

$$
W=e\left(X_{0}, X_{1}, \ldots, X_{n-1}, g_{\ell-1}\right)
$$

Observe that $W=g_{n+\ell-1}^{2^{n}-1}$.

- $\mathcal{B}$ gives $\alpha$ the public parameters $\left(W,\left\{X_{i}\right\}_{i \in[0, n+1]}, Y\right)$

- Now $\mathcal{A}$ is allowed to ask for private keys for users $u \notin S$ of Hamming weight $\ell$. $\mathcal{B}$ computes

$$
\mathrm{sk}_{u}=Z_{u}^{r} / \prod_{j \in S, j \neq u} Z_{2^{n}-1-j+u}
$$

Observe that

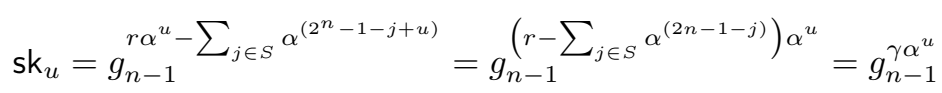

as desired. 
- When $\mathcal{A}$ asks for the challenge, $\mathcal{B}$ lets $\mathrm{Hdr}=\left(V, e\left(V, g_{n-1-\ell}\right)^{r}\right)$ and responds with $(\mathrm{Hdr}, K)$. Observe that

$$
e\left(V, g_{n-1-\ell}\right)^{r}=g_{n-1}^{r t}=g_{n-1}^{t\left(\gamma+\sum_{u \in S} \alpha^{\left(2^{n}-1-u\right)}\right)}
$$

which means $\left(V, e\left(V, g_{n-1-\ell}\right)^{r}\right)$ is a valid header for the set $S$. Also, observe that if $K=g_{n+\ell-1}^{t \alpha^{\left(2^{n}-1\right)}}$, then $K$ is the correct key for this header.

- When $\mathcal{A}$ returns a guess $b$ for which $K$ it is given, $\mathcal{B}$ returns $b$ as its guess.

As shown above, $\mathcal{B}$ perfectly simulates the view of $\mathcal{A}$ in the broadcast encryption security game. Therefore, $\mathcal{B}$ has the same advantage as $\mathcal{A}$, which must therefore be negligible, as desired.

\section{Our Third Construction}

In this section, we give our third and final broadcast scheme. This scheme is based on the basic broadcast scheme of Gentry and Waters [GW09], henceforth called the GW scheme. Like the BGW scheme, the GW scheme has public keys consisting of $O(N)$ elements, where $N$ is the number of users. Our idea is to, similar to Constructions 1 and 3 , run the GW scheme in the higher levels of a multilinear map, and derive the public key elements from $O(\log N)$ low-level elements.

However, unlike the BGW public parameters, which are all derived from a single scalar $\alpha \in \mathbb{Z}_{p}$, each of the GW public key elements are derived from a separate random scalar. Therefore, we cannot possibly hope to simulate the GW public key elements exactly. Instead, we we generate them using a NaorReingold-style PRF [NR97].

Also, unlike the BGW scheme, the secret keys in the GW scheme have $O(N)$ group elements. To make our scheme more efficient, and more importantly to make our scheme identity-based, we need to shrink the secret keys to $O(\log N)$ elements. To accomplish this, we observe that the secret key components are actually some of the outputs of another Naor-Reingold-style PRF, and we can allow the secret key holder to compute just those outputs by puncturing the PRF, similar to Boneh and Waters [BW13.

We now present out scheme:

Construction 5. Let Setup' be the setup algorithm for a multilinear map, where groups have order p. Our final identity-based broadcast scheme consists of the following algorithms:

$\operatorname{Setup}(n)$ Takes as input the length $n$ of identities. Run the setup algorithm for a multilinear map, Setup', to construct an $n+1$-linear map with parameters params'. Draw a random $\alpha \in \mathbb{Z}_{p}$. For $i=0, \ldots, n-1$ and $b=0,1$, draw random $\beta_{i, b} \in \mathbb{Z}_{p}$. The public key is

$$
\mathrm{pk}=\left(\text { params }^{\prime},\left\{X_{i, b}=g_{1}^{\beta_{i, b}}\right\}_{i \in[0, n-1], b \in\{0,1\}}, W=g_{n+1}^{\alpha}\right)
$$

For any user $\mathbf{u} \in\{0,1\}^{n}$, note that we can compute

$$
Z_{\mathbf{u}} \equiv g_{n}^{\prod_{i=1}^{n} \beta_{i, u_{i}}}=e\left(X_{1, u_{1}}, X_{2, u_{2}}, \ldots, X_{n, u_{n}}\right)
$$


KeyGen(params, $\left.\alpha,\left\{\beta_{i, b}\right\}, \mathbf{u}\right)$ Pick a random $r_{\mathbf{u}} \in \mathbb{Z}_{p}$. Let

$$
\begin{aligned}
U_{0}^{(\mathbf{u})} & =g_{1}^{r_{\mathbf{u}}} \\
U_{i}^{(\mathbf{u})} & =X_{i, 1-u_{i}}^{r_{\mathbf{u}}}=g_{1}^{r_{\mathbf{u}} \beta_{i, 1-u_{i}}} \text { for } i=1, \ldots, n \\
U_{n+1}^{(\mathbf{u})} & =g_{n}^{\alpha} Z_{\mathbf{u}}^{r_{\mathbf{u}}}=g_{n}^{\alpha+r_{\mathbf{u}} \cdot \prod_{i=1}^{n} \beta_{i, u_{i}}}
\end{aligned}
$$

The secret key for user $\mathbf{u}$ is $\mathbf{s k}_{\mathbf{u}}=\left\{U_{i}^{(\mathbf{u})}\right\}_{i \in[0, n+1]}$.

Observe that for $\mathbf{v} \neq \mathbf{u}$, we can compute $Z_{\mathbf{v}}^{r_{\mathbf{u}}}$ by finding an $i^{*}$ where $v_{i^{*}}=$ $1-u_{i^{*}}$, and computing

$$
\begin{aligned}
e\left(X_{1, v_{1}}, \ldots, X_{i^{*}-1, v_{i^{*}-1}}, U_{i^{*}}^{(\mathbf{u})}, X_{i^{*}+1, v_{i^{*}+1}}, \ldots, X_{n, v_{n}}\right) & =g_{n}^{r_{\mathbf{u}} \beta_{i^{*}, v_{i}}} \cdot \prod_{i \neq i^{*}} \beta_{i, v_{i}} \\
& =g_{n}^{r_{\mathbf{u}} \cdot \prod_{i=1}^{n} \beta_{i, v_{i}}}=Z_{\mathbf{v}}^{r_{\mathbf{u}}}
\end{aligned}
$$

Enc(params, $S$ ) Choose a random $t \in \mathbb{Z}_{p}$ and compute the key and header as

$$
K=W^{t}=g_{n+1}^{t \alpha} \quad \text { and } \mathrm{Hdr}=\left(g_{1}^{t},\left(\prod_{\mathbf{u} \in S} Z_{\mathbf{u}}\right)^{t}\right)=\left(g_{1}^{t}, g_{n}^{t \sum_{\mathbf{u} \in S} \prod_{i=1}^{n} \beta_{i, u_{i}}}\right)
$$

where $Z_{\mathbf{u}}$ are computed as above.

$\operatorname{Dec}\left(\right.$ params, $\left.\mathbf{u}, \mathrm{sk}_{\mathbf{u}}, S, \mathrm{Hdr}\right)$ If $\mathbf{u} \notin S$, output $\perp$. Otherwise, write $\mathrm{Hdr}=\left(C_{0}, C_{1}\right)$. Compute

$$
k=\frac{e\left(U_{n+1}^{(\mathbf{u})} \cdot \prod_{\mathbf{v} \in S, \mathbf{v} \neq \mathbf{u}} Z_{\mathbf{v}}^{r_{\mathbf{u}}}, C_{0}\right)}{e\left(U_{0}^{(\mathbf{u})}, C_{1}\right)}
$$

Observe that if $\left(C_{0}, C_{1}\right)$ are as above, we can write $k$ as $g_{n+1}^{c}$ where

$$
c=\left(\alpha+r_{\mathbf{u}} \prod \beta_{i, u_{i}}+\sum_{\mathbf{v} \in S, \mathbf{v} \neq \mathbf{u}} r_{\mathbf{u}} \prod \beta_{i, v_{i}}\right) \cdot t-r_{\mathbf{u}} \cdot\left(t \sum_{\mathbf{v} \in S} \prod \beta_{i, v_{i}}\right)=\alpha t
$$

as desired.

Correctness follows from the comments above.

Differences from $G W$. In the Gentry and Waters scheme [GW09], the $Z_{\mathbf{u}}$ are generated independently and given explicitly in the public parameters (as elements of the source group $\mathbb{G}_{1}$ ). In our scheme, the $Z_{\mathbf{u}}$ are generated pseudorandomly by means of a Naor-Reingold PRF. Similarly, in the GW scheme, the $Z_{\mathbf{v}}^{r_{\mathbf{u}}}$ for $\mathbf{v} \neq \mathbf{u}$ are also given explicitly to user $\mathbf{u}$. In our scheme, we note that the $Z_{\mathbf{v}}^{r_{\mathbf{u}}}$ for fixed $\mathbf{u}$ actually form another Naor-Reignold PRF, which we puncture at the point $\mathbf{u}$ to allow user $\mathbf{u}$ to compute the necessary values without learning $Z_{\mathbf{u}}^{r_{\mathbf{u}}}$. Our puncturing follows the puncturing used by Boneh and Waters BW13].

Comparison to Constructions 1 and [3. Construction 5 has a couple advantages and disadvantages over our previous schemes: 
- Unlike the BGW-based schemes, there are no high-degree terms being generated. This means we do not need the secret parameters for the multilinear map to set up our scheme. Therefore, we can use a map from some trusted third party. We do, however, need to make sure re-randomization parameters are available in the groups $\mathbb{G}_{1}$ and $\mathbb{G}_{n}$ to re-randomize the header elements. If we are using a map that we did not set up, we also need to re-randomize the user secret keys.

- To handle identities of length $\lambda$, the total multilinearity of Construction 5 is $\lambda+1$. Compare this to $2 \lambda$ and $1.440\left(\lambda+\left(\log _{2} \lambda\right) / 2\right)$ from the previous constructions.

- On the negative side, secret keys in Construction [5 consist of $O(\log N)$ group elements, compared to the single element secret keys of the previous schemes.

- For security, we unfortunately are unable to prove security relative to a non-interactive assumption. In the original GW scheme, the security proof involved manipulating the $Z_{\mathbf{u}}$ for $\mathbf{u} \notin S$. Since each of the $Z_{\mathbf{u}}$ are independent in the GW scheme, this is achievable. For our scheme, however, the $Z_{\mathbf{u}}$ are generated from $O(\log N)$ parameters, meaning we cannot modify them independently. Instead, in the full version BWZ14, we opt to prove security in the generic multilinear map model. We note, however, that we obtain a better generic security theorem than is possible for Constructions 1 and 3

Acknowledgments. This work is supported by NSF, DARPA, IARPA, and others, as listed in the full version.

\section{References}

[BBW06] Barth, A., Boneh, D., Waters, B.: Privacy in encrypted content distribution using private broadcast encryption. In: Di Crescenzo, G., Rubin, A. (eds.) FC 2006. LNCS, vol. 4107, pp. 52-64. Springer, Heidelberg (2006)

[BGI $\left.{ }^{+} 01\right]$ Barak, B., Goldreich, O., Impagliazzo, R., Rudich, S., Sahai, A., Vadhan, S.P., Yang, K.: On the (Im)possibility of Obfuscating Programs. In: Kilian, J. (ed.) CRYPTO 2001. LNCS, vol. 2139, p. 1. Springer, Heidelberg (2001)

[BGW05] Boneh, D., Gentry, C., Waters, B.: Collusion resistant broadcast encryption with short ciphertexts and private keys. In: Shoup, V. (ed.) CRYPTO 2005. LNCS, vol. 3621, pp. 258-275. Springer, Heidelberg (2005)

[BS03] Boneh, D., Silverberg, A.: Applications of multilinear forms to cryptography. Contemporary Mathematics 324, 71-90 (2003)

[BW13] Boneh, D., Waters, B.: Constrained pseudorandom functions and their applications. In: Sako, K., Sarkar, P. (eds.) ASIACRYPT 2013, Part II. LNCS, vol. 8270, pp. 280-300. Springer, Heidelberg (2013)

[BWZ14] Boneh, D., Waters, B., Zhandry, M.: Low overhead broadcast encryption from multilinear maps. Full version available at the Cryptology ePrint Archives, Report 2014/195

[BZ14] Boneh, D., Zhandry, M.: Multiparty key exchange, efficient traitor tracing, and more from indistinguishability obfuscation. In: Garay, J.A., Gennaro, R. (eds.) CRYPTO 2014, Part I. LNCS, vol. 8616, pp. 480-499. Springer, Heidelberg (2014)

[CLT13] Coron, J.-S., Lepoint, T., Tibouchi, M.: Practical multilinear maps over the integers. In: Canetti, R., Garay, J.A. (eds.) CRYPTO 2013, Part I. LNCS, vol. 8042, pp. 476-493. Springer, Heidelberg (2013) 
[Del07] Delerablée, C.: Identity-Based Broadcast Encryption with Constant Size Ciphertexts and Private Keys 2, 200-215 (2007)

[DF02] Dodis, Y., Fazio, N.: Public Key Broadcast Encryption for Stateless Receivers. In: Feigenbaum, J. (ed.) DRM 2002. LNCS, vol. 2696, pp. 61-80. Springer, Heidelberg (2003)

[DF03] Dodis, Y., Fazio, N.: Public key trace and revoke scheme secure against adaptive chosen ciphertext attack. In: Desmedt, Y.G. (ed.) PKC 2003. LNCS, vol. 2567, pp. 100-115. Springer, Heidelberg (2002)

[DPP07] Delerablée, C., Paillier, P., Pointcheval, D.: Fully collusion secure dynamic broadcast encryption with constant-size ciphertexts or decryption keys. In: Takagi, T., Okamoto, T., Okamoto, E., Okamoto, T. (eds.) Pairing 2007. LNCS, vol. 4575, pp. 39-59. Springer, Heidelberg (2007)

[FHPS13] Freire, E.S.V., Hofheinz, D., Paterson, K.G., Striecks, C.: Programmable Hash Functions in the Multilinear Setting. In: Canetti, R., Garay, J.A. (eds.) CRYPTO 2013, Part I. LNCS, vol. 8042, pp. 513-530. Springer, Heidelberg (2013)

[FN94] Fiat, A., Naor, M.: Broadcast Encryption. In: Stinson, D.R. (ed.) CRYPTO 1993. LNCS, vol. 773, pp. 480-491. Springer, Heidelberg (1994)

[FP12] Fazio, N., Perera, I.M.: Outsider-Anonymous Broadcast Encryption with Sublinear Ciphertexts. In: Fischlin, M., Buchmann, J., Manulis, M. (eds.) PKC 2012. LNCS, vol. 7293, pp. 225-242. Springer, Heidelberg (2012)

[GGH13a] Garg, S., Gentry, C., Halevi, S.: Candidate multilinear maps from ideal lattices. In: Johansson, T., Nguyen, P.Q. (eds.) EUROCRYPT 2013. LNCS, vol. 7881, pp. 1-17. Springer, Heidelberg (2013)

$\left[\mathrm{GGH}^{+} 13 \mathrm{~b}\right]$ Garg, S., Gentry, C., Halevi, S., Raykova, M., Sahai, A., Waters, B.: Candidate indistinguishability obfuscation and functional encryption for all circuits. In: Proc. of FOCS 2013 (2013)

[GST04] Goodrich, M.T., Sun, J.Z., Tamassia, R.: Efficient tree-based revocation in groups of low-state devices. In: Franklin, M. (ed.) CRYPTO 2004. LNCS, vol. 3152, pp. 511-527. Springer, Heidelberg (2004)

[GW09] Gentry, C., Waters, B.: Adaptive security in broadcast encryption systems (with short ciphertexts). In: Joux, A. (ed.) EUROCRYPT 2009. LNCS, vol. 5479, pp. 171-188. Springer, Heidelberg (2009)

[HS02] Halevy, D., Shamir, A.: The lsd broadcast encryption scheme. In: Yung, M. (ed.) CRYPTO 2002. LNCS, vol. 2442, pp. 47-60. Springer, Heidelberg (2002)

[LPQ12] Libert, B., Paterson, K.G., Quaglia, E.A.: Anonymous broadcast encryption: Adaptive security and efficient constructions in the standard model. In: Fischlin, M., Buchmann, J., Manulis, M. (eds.) PKC 2012. LNCS, vol. 7293, pp. 206-224. Springer, Heidelberg (2012)

[LSW10] Lewko, A.B., Sahai, A., Waters, B.: Revocation systems with very small private keys. In: IEEE Symposium on Security and Privacy, pp. 273-285 (2010)

[NNL01] Naor, D., Naor, M., Lotspiech, J.: Revocation and tracing schemes for stateless receivers. In: Kilian, J. (ed.) CRYPTO 2001. LNCS, vol. 2139, pp. 41-62. Springer, Heidelberg (2001)

[NP00] Naor, M., Pinkas, B.: Efficient trace and revoke schemes. In: Frankel, Y. (ed.) FC 2000. LNCS, vol. 1962, pp. 1-20. Springer, Heidelberg (2001)

[NR97] Naor, M., Reingold, O.: Number-theoretic constructions of efficient pseudo-random functions. In: FOCS, pp. 458-467 (1997)

[SF07] Sakai, R., Furukawa, J.: Identity-Based Broadcast Encryption. IACR Cryptology ePrint Archive (2007) 